\title{
A single patient study of high dose isoflavones to enhance gemzar chemotherapy in post resection and post chemoradiation adjuvant treatment of pancreatic adenocarcinoma
}

\author{
Adam J. Nehr III ${ }^{*}$ and Barbara J. Nehr ${ }^{2}$ \\ ${ }^{1}$ Retired Instrumentation Camera Specialist, Kennedy Space Center, USA \\ ${ }^{2}$ Retired Financial analyst, SAIC, USA
}

\begin{abstract}
Even in stage 2 presentations, pancreatic adenocarcinoma is considered a deadly disease. Overall from all stages only $7 \%$ of the patients survive for five years and almost all of those are from the lower stage presentations (stage $0-2 \mathrm{~b}$ which represent $25 \%$ of all patients) and are also eligible for resection. In this population, only $25 \%$ survive. The causes of this dismal statistic are theorized to be the pancreatic cancer cells' propensity to form a protective stroma which blocks the action of the most commonly used chemotherapy agents and also, the continued activation of protein pathways, specifically NF- $\mathrm{B}$ that results in an inhibition of apoptosis. A recent study of high dose genistein in orthotopic mouse models indicated that this novel therapy may both retard stroma formation to a permeable level and also deactivate the Notch NF- $\mathrm{B}$ pathway leading to increased apoptosis and the prevention of recurring disease. The case presented here is of a single patient given this novel treatment at full dosing as defined by the orthotopic study during the Gemzar phase of chemotherapy and continuing at a maintenance dose afterward for a period of five years. The patient survived with remarkably lower miR-21 and CA19-9 expression levels and no recurring disease. She remains disease free as of the date of this paper five years and ten months post resection. Thus the authors believe that further study of this effect is warranted.
\end{abstract}

\section{Introduction}

In stage $2 \mathrm{a}$ pancreatic adenocarcinoma, pylorus-preserving pancreaticoduodenectomy (PPPD, an enhanced Whipple procedure) is usually done with curative intent. It is successful in less than $25 \%$ of the cases. To inhibit local or distant recurrence, surgery is commonly followed by a sequence of chemo-radiation using capecitabine (Zeloda) and a separate sequence of straight chemotherapy using gemcitabine (Gemzar); recent developments have now extended treatment options to include chemotherapy regimens such as FOLFIRINOX or Gemcitabine-Abraxane. The protocols used to deliver these treatment elements vary by the experience of the administering physician and the procedures currently recognized by the medical community.

Recent studies detailing the variables that factor into survival indicate that five year survival of $25 \%$ or less of patients in this overall category of resectable disease, irrespective of staging and treatment, is expected with the standard of care protocols as they are currently used [1]. The overall five year survival of all pancreatic cancer patients from all stages of diagnosis is about $5 \%$; this group is primarily composed of patients who are eligible for and have undergone resection and the above described treatment.

The current low survival rate reflects the propensity of pancreatic adenocarcinoma micro-metastatic cells 1) to spread undetected through perineural or other routes of tissue invasion undetectable by the surgical team and 2) to develop resistance to chemotherapy drugs. Such dual characteristics allow the disease to recur in a high percentage of otherwise successful resection cases. The dual phase adjuvant treatment is intended to correct the vectors of recurrence but it is not universally successful. This is due to the well-known proclivity of pancreatic cancer cells to rapidly develop a protective stroma which imparts confer resistance to chemo/radiation treatments and to the Gemzar, a blood-borne agent used for the straight chemotherapy phase. In most cases, micro-metastatic cells remain in the surgical field, in the nerve proteins, or even in the tissue margins. However, these cells are undetectable after resection and adjuvant therapy. When cells are no longer inhibited by the adjuvant therapy, which cannot be continued indefinitely due to toxicity, disease recurs in a high percentage of patients.

Recently published works indicate that the continued activation of signaling pathway NF- $\kappa \mathrm{B}$ is a prime factor in disease recurrence [24]. The signaling pathway becomes active at the onset of the original disease and remains active after surgical intervention unless inhibited by some biochemical means.

It is theorized that this may cause the disease to arise from cells that were healthy at the time of resection but which become malignant because the mechanism that caused the disease in the first place is still active, and thus, capable of affecting healthy cells in a manner identical

Correspondence to: Adam Nehr, 20448 Majestic Street, Orlando Florida 32833 3921, USA, Tel: 407-568-3197/407-694-1392; E-mail: gnur@gnc.net

Key words: pancreatic cancer, single patient study, microRNA, isoflavone, Gemzar, CA19-9

Received: February 05, 2016; Accepted: February 22, 2016; Published: February 25,2016 
to that which created the original tumor. While there is some evidence that this mechanism is more complex than as stated above [1] in cancer overall, the data for pancreatic adenocarcinoma appears to favor the notion that inhibition of NF- $\mathrm{KB}$ would be beneficial to patient survival.

Recent studies by Wayne State University detailed the use of high dose isoflavones, derived from soy beans, to achieve increased apoptosis during Gemzar treatment, in part due to inactivation of NF- $\kappa B$ signaling [2]. However, in addition to NF- $\kappa B$ interruption, the mechanism is suspected to be a broad spectrum inhibitor to some if not all protein kinase signaling involved in disease initiation and progression.

The addition of high dose isoflavones to the current standard-ofcare treatment has potential to provide a novel enhancement in the anti-tumor activity of the chemotherapy delivered to micro-metastatic cells that were undetectable within the operative field or elsewhere in the body during or immediately after surgery. In vitro and in vivo preclinical studies also indicate that isoflavones inactivates NF- $\kappa B$ signaling and a broad spectrum of protein kinases, which in turn theoretically inhibit disease recurrence by deactivating the primary signaling pathway or pathways essential to disease progression.

The objective of the current work is to describe the case history of a patient who has survived five years post-diagnosis of pancreatic cancer. The study details the use of isoflavones utilized in an effort to realize the curative intent of the surgery with more certainty than that provided by current standard-of-care surgical and adjuvant therapeutic protocols alone.

\section{Materials and methods}

\section{Patient history, diagnosis and initial treatment}

This history, except where noted, is presented in chronological sequence with each component in the order that it presented clinically. The study lasts for a full five year period starting at post resection and ending at the 5 year examination and imaging study. The patient is a 65 year old Caucasian female who presented February 27 2010, with significantly elevated aspartate aminotransferase (AST) at 151, alanine aminotransferase (ALT) at 242, alkaline phosphatase (ALP) at 682, moderately elevated total bilirubin (2.0), pruritis, and subsequent jaundice. No abdominal discomfort was indicated. Test results and symptoms are classical indicators of pancreatic cancer [5], but can also indicate several less dire diseases.

The patient revealed that her maternal grandfather had succumbed to breast cancer and that a maternal uncle had succumbed to adenocarcinoma of the pancreas. She had no known genetic connection to the Ashkenazi Jewish population. All of these characteristics are classic factors indicative of a genetic predisposition to this disease; this particular patient was positive for two. She was also a long term moderate smoker who had quit 2 years prior to disease onset.

Abdominal ultrasound revealed a mild dilation of the common bile duct and a vague indication of a mass in the pancreatic head. To confirm the suspected diagnosis of malignancy, an MRI was performed, but it was unrevealing except to confirm the dilation of the bile duct. A spiral CT scan and endoscopic ultrasound also confirmed the dilation but were otherwise unrevealing.

A CA19-9 blood test indicated moderate elevation (66) and as a result, an endoscopic ultrasound was ordered. The endoscopic ultrasound test however proved to be unrevealing of malignancy and no biopsy was taken.
A highly specialized consultation was sought to affect a firm diagnosis. A high volume research and teaching facility was selected, based on the availability of a surgeon with extensive experience with the Whipple procedure, a known benefit to survival [1]. Another consideration was that most of the procedures done by this surgeon were performed, when possible, laparoscopically. A final factor in the choice of facility was the presence of exceptionally advanced research and diagnostic capabilities able to use cutting edge methods to accurately confirm the suspected diagnosis of adenocarcinoma of the pancreatic head. Tests performed at this facility were (in order) MRI with contrast, endoscopic ultrasound with stenting, a second CA19-9 (now 600) and finally an endoscopic retrograde cholangiopancreatography (ERCP) with needle biopsy. The combined findings of these tests confirmed the presence of a $2 \mathrm{~cm}$ adenocarcinoma of the pancreatic head.

Surgery was performed laparoscopically on April 2, 2010, one day after the ERCP/needle biopsy, by a surgeon specializing in the procedure who performs over 100 procedures per year. He was assisted by several resident surgeons.

The total time from the discovery of elevated blood AST and ALT to the diagnosis and subsequent surgery was 34 days. Less than 200 $\mathrm{ml}$ of blood loss was recorded during the surgery. Low blood loss is considered by some surgeons to be an enhancement to survival statistics and was facilitated by the non-invasive method used. Frozen sections of the tissue margins adjacent to the operative field were analyzed before surgical reconstruction began. Microscopic examination of the frozen sections confirmed that all surgical margins and all nine harvested lymph nodes were clear of detectable micro-metastatic cells.

The surgery overall lasted 10 hours. Post operative patient comfort was greatly enhanced by the laparoscopic versus open procedure and she was walking, assisted, the next day. She experienced minimal post operative side effects and was released from the hospital six days post procedure.

Subsequent in-vitro histological examination of all specimens removed confirmed clear margins all around and the absence of lymph node metastasis. The tumor mass was moderately well differentiated and $2 \mathrm{~cm}$ in size over its largest dimension. Collectively the findings confirmed the diagnosis of stage 2 a disease.

\section{Materials}

\section{Zeloda, isoflavones, and gemzar}

All chemotherapy agents were of standard composition.

\section{Zeloda}

The dosing of Zeloda during the radiation phase was $1500 \mathrm{mg}$ twice daily, and was not adjusted during the treatment because the patient tolerated the maximum dose. This is standard treatment and has been classically used in this manner for many years.

\section{Isoflavones}

The isoflavones (calculated based on genistein content) dosage was derived by a body mass calculation using a target of $27 \mu \mathrm{mol} /$ $\mathrm{L}$ in blood plasma and using a formula from the Wayne State study [3] which indicated that an isoflavone (genistein) dose of $16 \mathrm{mg} / \mathrm{kg}$ of body weight would yield the desired serum concentration. When the test began the subject weighed $77 \mathrm{~kg}$, resulting in a dose of 1,264 $\mathrm{mg} /$ day or 13 capsules per day in three doses of 5/3/5 capsules, respectively. The isoflavone (genistein) was supplied in the form of a 
Nehr AJ (2016) A single patient study of high dose isoflavones to enhance gemzar chemotherapy in post resection and post chemoradiation adjuvant treatment of pancreatic adenocarcinoma

product from Life Extensions (Ultra Soy), delivered in capsule form. Each capsule contained $93.75 \mathrm{mg}$ Genistein and $87.5 \mathrm{mg}$ of Daidzein. After the Gemzar treatments ended, the higher dosage of isoflavones was continued for three months, after which the dosage was reduced to $55 \%$ (in three doses per day $3 / 2 / 3$ capsules respectively for a total of eight) of the dose indicated by the Wayne State study [3] and then in 2013 it was further reduced to $44 \%$ of the full dosage as indicated by the Wayne State Paper. Since the Gemzar was no longer on board the $44 \%$ dosage was theorized to be sufficient to inhibit the protein signaling. The exact threshold for this effect needs to be studied further in larger populations and also in the laboratory so a minimum effective dose can be determined.

Due to the manufacturer discontinuing the Ultra Soy product in mid 2013 a substitute was found in the form of pure genistein, (no Daidzein in formulation) produced by Vital Nutrients, which contains $125 \mathrm{mg}$ per capsule of genistein. The dosing was adjusted in mid 2013 to reflect the subject's now $53 \mathrm{~kg}$ body mass and the dose rounded down to three $125 \mathrm{mg}$ capsules per day, (a 50\% dose would equal 424 $\mathrm{mg}$; this dose equaled $44 \%$ ) given in a morning dose of one capsule and an evening dose of two capsules, resulting in an overall daily genistein dose of $9.43 \mathrm{mg} / \mathrm{kg}$ of body weight. The reduction to $44 \%$ was in response to the supply of the genistein isoflavone being limited and the necessity to extend the supply as long as possible. The supply is limited but sustainable at this level. This regimen continues as of the date of this paper, five years from surgery.

\section{Gemzar}

Standard dosing for this agent is calculated by determining the patient's body surface area by formula (Du Buois \& DuBuois, Gehan, Haycock, or Mosteller method) The patient at time of initial treatment was approx $165 \mathrm{~cm}$ tall and $77 \mathrm{~kg}$ in mass. By Dubois and Dubois method this resulted in a 1.84 factor. The standard dosing regimen recommended by the drug maker is $1000 \mathrm{mg}$ multiplied by the factor for initial treatment. The result was a dose of $1840 \mathrm{mg}$ per session delivered intravenously by power port infusion. This dose was consistent but as detailed later the frequency of treatment was altered to compensate for platelet level concerns (Figure 1).

\section{MicroRNA-21 (miR-21)}

\section{Collection of plasma and RNA isolation}

The collection of biospecimen was through personal interest of the patient and it was not subjected to any clinical protocol. A research team at Wayne State University volunteered to meet the personal request from the patient for conducting measurement of miR-21 expression level.

Blood samples, including plasma, were collected from the sole cancer patient and synchronized with imaging studies for a period of about three years; plasma was isolated. Total RNA containing small RNA was isolated from the plasma of ten healthy subjects, and also from the plasma of the single cancer patient, each time using Trizol LS reagent (Life Technologies) according to the manufacturer's protocol with the following modifications. The Trizol LS reagent was added to plasma in the ratio of 3:1, incubated for 5 minutes and mixed with chloroform. After centrifugation the upper aqueous phase was transferred to a new tube and isopropanol was added and incubated for 30 minutes followed by centrifugation at $12,000 \mathrm{x}$ g for 10 minutes. The precipitate was washed with $75 \%$ ethanol and centrifuged at 7,500 for 5 minutes. The RNA pellet was eluted in water in a final volume of $20 \mu \mathrm{l}$.

\section{Formalin-fixed paraffin-embedded (FFPE) tissue}

Pancreatic tumor tissue (tumor sample) and tissue that was anatomically farther away from the pancreatic tumor (normal sample) was fixed using formalin and embedded in paraffin using standard protocol. Hematoxylin and eosin staining was used for histological confirmation of cancer. Total RNA was isolated using RNeasy Kit (QIAGEN) according to the manufacturer's protocol with some modifications. Four tissue sections of $10 \mu \mathrm{m}$ thick from both normal

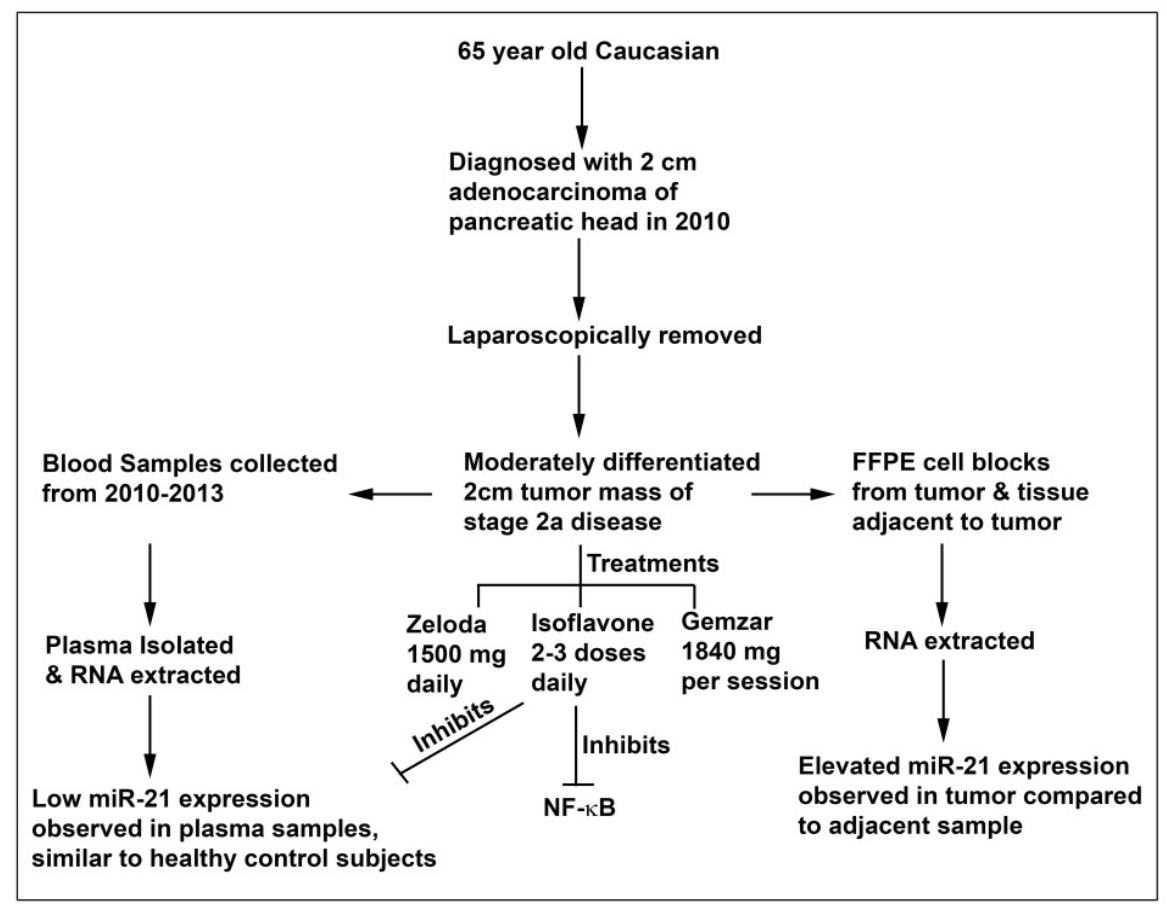

Figure 1. Graphical abstract of experimental procedure in patient. 
and tumor tissue were placed in micro tubes along with $1 \mathrm{ml}$ xylene. After vortexing for 30 seconds, samples were centrifuged for 2 minutes at room temperature.

One $\mathrm{ml}$ of ethanol was added to the pellet, centrifuged for 2 minutes, and was resuspended in $240 \mu \mathrm{l}$ of Buffer PKD along with $10 \mu \mathrm{l}$ of proteinase $\mathrm{K}$ and incubated at $55^{\circ} \mathrm{C}$ for 15 minutes, and then at $80^{\circ} \mathrm{C}$ for 15 minutes. Buffer RBC along with $1200 \mu$ of ethanol was added to the aliquot that was subsequently applied directly to the RNeasy column. RNA was washed with buffer solution to remove impurities and eluted in a final volume of $25 \mu \mathrm{l}$.

TaqMan miRNA Real-Time Reverse Transcriptase-Polymerase Chain Reaction (RT-PCR)

Quantitative RT-PCR (qRT-PCR) provides quantitative analysis of microRNA (miRNA) expression in real time for the miRNAs of interest. The RNA isolated from plasma of 10 normal subjects were pooled together and used as a control to compare against the single cancer patient. The isolated RNA, both from plasma and from FFPE tissue samples were reverse transcribed using TaqMan MicroRNA Assay kit (Applied Biosystems) to study the expression of miR-21. About $10 \mathrm{ng}$ of RNA from either plasma samples or FFPE tissue samples were used along with $7 \mu$ of master mix containing dNTPs, reverse transcriptase and RNase inhibitor and $3 \mu \mathrm{l}$ of respective primer. The mixture was incubated at $16^{\circ} \mathrm{C}$ for 30 minutes, $42^{\circ} \mathrm{C}$ for 30 minutes, followed by $85^{\circ} \mathrm{C}$ for 5 minutes. Real-time PCR reactions were then carried out in a total volume of $10 \mu \mathrm{l}$ reaction mixture containing $5 \mu \mathrm{L}$ of $2 \mathrm{X}$ Taqman Universal PCR master mix, $3.8 \mu \mathrm{l}$ of water, $0.7 \mu \mathrm{l}$ of RT product and $0.5 \mu \mathrm{l}$ of probe. All reactions, including controls were performed in triplicate using StepOnePlus Real-Time PCR (Applied Biosystems). Relative expression of miRNAs was analyzed using Ct method and was normalized by RNU48 expression.

\section{Data directly related to apparent benefits, apoptosis} and NF- $\kappa B$ inactivation

\section{miR-21 expression and implications}

One aspect of treating pancreatic ductal adenocarcinoma is that there is no definitive early test capable of identifying the disease in its early stages. The bulk of PC patients present with stage 4 metastatic disease. At that point of presentation, surgery with curative intent is frequently impossible. This is due to tumor involvement with the portal vein and mesenteric artery or because so many other organs and areas of the abdomen are invaded. Even in patients with a known family history or genetic precursors that would make them candidates for periodic screening, testing for early stage disease is impossible due to lack of a reliable test procedure. Such limitations also inhibit the research being conducted here because there is no accepted method to measure efficacy of any individual treatment given. Only survival and sparse statistical data related to miR-21 and CA19-9 levels are available and those tests are not always revealing.

The over-expression of miR-21 is known to occur in pancreatic adenocarcinoma and is being considered as a possible metric for early detection. The patient in this study was diagnosed at stage 2a because of the location of the tumor and its effect on the bile flow. The tumor fortuitously blocked the flow of bile causing elevated AST, ALT, and bilirubin levels.

Investigating these elevated levels was complex and accurate diagnosis finally confirmed immediately prior to surgery by the return of a fine needle biopsy done during an ERCP.
Testing of miR-21 expression is theorized to be revealing at the early phase of this disease [6-8], rendering this metric potentially valuable for study. The patient was not tested for miR-21 expression prior to surgery but tissue blocks from the removed tumor were preserved and tested at the start of the genistein/Gemzar phase of treatment. The classic test method for indicating possible pancreatic adenocarcinoma is the carbohydrate antigen test CA19-9 which is a sialylated Lewis antigen marker. This test is unfortunately reactive to broad inflammatory processes as well and, thus, produces many false positives.

Also, in Lewis negative patients, the CA19-9 test fails to produce a positive indication when disease is actually present. Thus while useful as a broad indicator, it is not considered a conclusive test for pancreatic cancer. That said, it is the current standard of care indicator to assess latent or recurring disease. In the case of the patient who is the subject of this paper, both tests were run concurrently to allow a comparative study that could be referenced to the imaging studies.

The actual study comprised a two and one half year period from six months post surgery (10/05/2010, the start of the Gemzar treatment) to the end of a three year period in $(5 / 24 / 2013)$. The study was discontinued because it was found that miR-21 expression level was reactive to inflammation but was also persistently and uncharacteristically low in this patient when compared with a large body of post-resection pancreatic adenocarcinoma patients. Figure 2 correlates CA19-9 with comparative miR-21 expression with reference to the normal healthy patient levels of each and with this patient's data expressed over time.

\section{Results of miR-21 v CA19-9}

Figure 2 displays the comparative expression of miR-21 for a period of two and one half years, taken from plasma samples. The top horizontal limit line shows the average post resection expression of the post resection patients in the Wayne State study [8] and the lower line is the normal for healthy cancer free subjects, below which none of the patients in the Wayne state study ever returned. Note that the subject in this study has moved below the line except in times of liver inflammation (Figure 3).

The expression levels of miR-21 from the control group of normal subjects were compared against the single cancer patient for a period of about three years. The miR-21 is an oncogenic miRNA in pancreatic cancer. In fact, it is considered as one of the globally over-expressed miRNA in many cancers. Based on this fact, the expression level of miR-21 from the single cancer subject should have been significantly higher than normal subjects. In contrast, the expression level of miR21 was very similar to normal subjects for most of the two and one half years tested except for four times where it was higher compared to normal subjects, as shown in figure 3 . These elevations correlated with periods of raised liver numbers caused by a liver to diaphragm adhesion problem (described later in this paper) but were far lower even at their highest point than most post resection patients in the cited study [8].

\section{Comparative expression of miR-21 from normal and tumor FFPE samples}

The expression level of miR-21 was compared between normal and tumor FFPE tissue sample from the single patient. Unlike plasma samples the expression of miR-21 was significantly higher than the adjacent tissue from the same patient, as shown in Figure 4.

During this three year period paired blood samples were drawn; from each draw one sample was sent to the lab at MD Anderson Cancer 
Nehr AJ (2016) A single patient study of high dose isoflavones to enhance gemzar chemotherapy in post resection and post chemoradiation adjuvant treatment of pancreatic adenocarcinoma

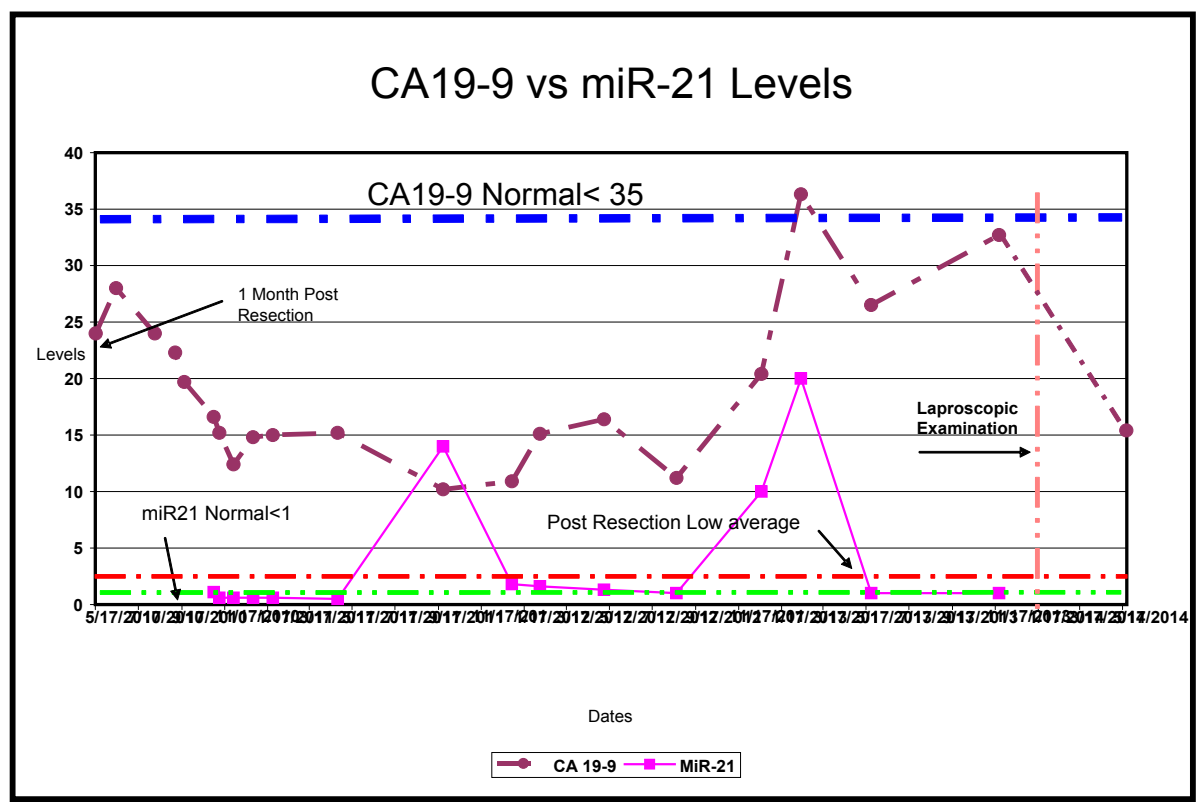

Figure 2. Correlation of CA19-9 with miR-21 expression with reference to the normal healthy patients.

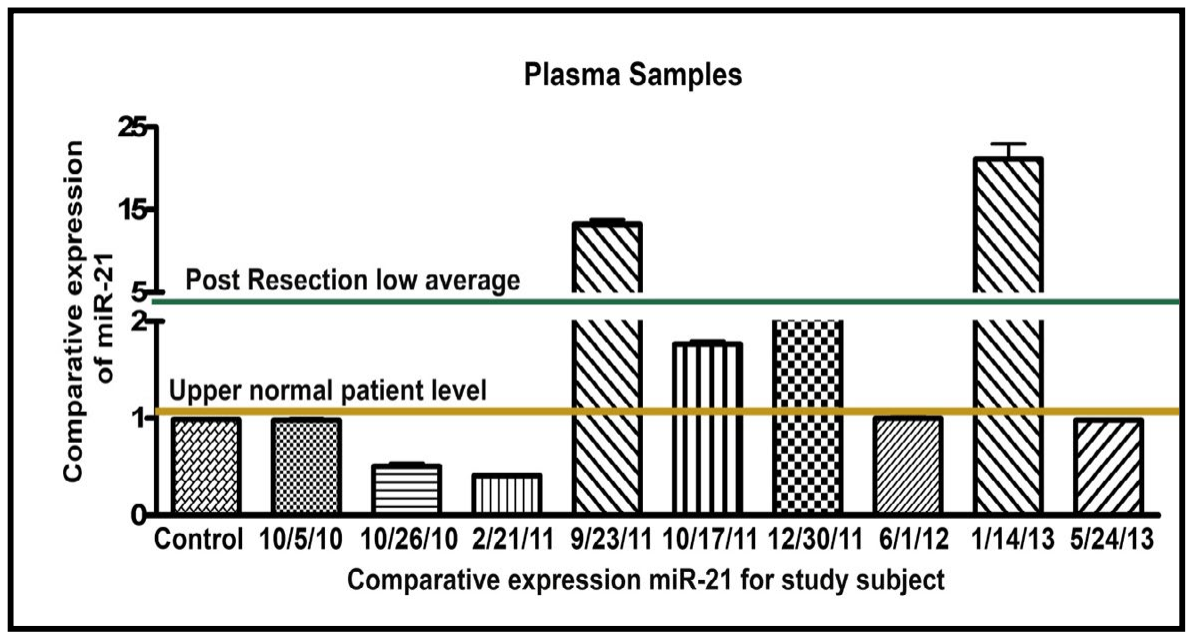

Figure 3. Comparative experession of miR-21 from plasma samples for a period of more than 2 years with healthy cancer=free subjects.

Center in Orlando, Florida for CA19-9 testing and the second plasma sample was shipped in a citrate tube surrounded by dry ice to Karmanos Cancer Center at Wayne State University for measurement of miR21 expression. The miR-21 testing took approximately one month to complete, so the dates for these tests must be offset one month to the right for velocity comparison purposes. The composite chart (Figure 1 above) is not offset and reflects the reporting dates for miR-21 study. The date for the CA19-9 data is offset one day from collection since reporting on that test is 24 hours. Figure 1 appears to reveal that miR-2 1 expression tracks inflammatory rises as does CA19-9 but is a predictor not a reactor, at least in this patient.

Over the entire time that the data shown was produced, the patient remained cancer free as indicated by imaging studies, symptomology, and a laparoscopic examination performed on 13 August 2013.

\section{Subsequent patient history}

This section is provided to aid subsequent research in identifying possible side effects and to give a single patient baseline to look for consistencies in larger populations.

\section{Continuation of genistein post-gemzar chemotherapy}

\section{Adjuvant treatment and side effects}

After a recovery period of eight weeks after surgery, chemoradiation therapy was initiated, consisting of daily ingestion of Zeloda (capecitabine), and CT guided radiation administered 5 days a week for 5.5 weeks for a total of 28 treatments. A dose of 55 Gy was used per treatment. Whether because of radiation exposure or a reaction to the Zeloda, this phase of adjuvant treatment had significant side effects most notably disorientation, extreme fatigue, mild hair loss, loss of appetite and sense of taste resulting in weight loss, dry mouth, and extremely dry skin especially on the feet.

The patient completed the entire course of chemo-radiation. No hospitalization was required. Recovery was rapid and after a three week 
Nehr AJ (2016) A single patient study of high dose isoflavones to enhance gemzar chemotherapy in post resection and post chemoradiation adjuvant treatment of pancreatic adenocarcinoma

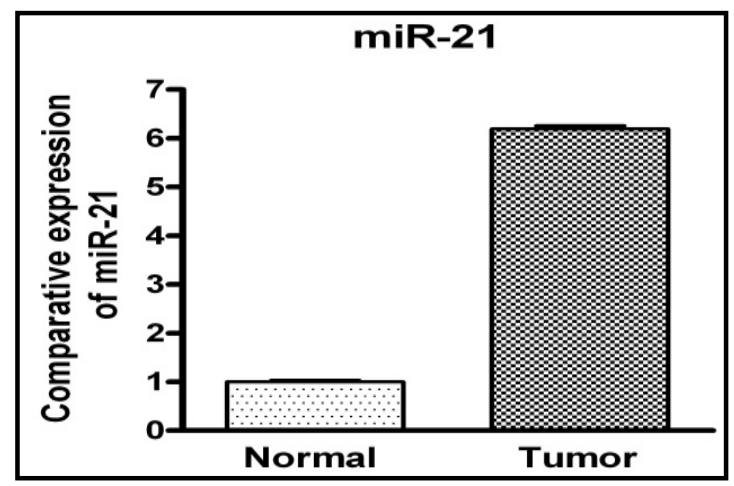

Figure 4. Comparative expression of miR-21 from normal and tumor formalin-fixed paraffin-embedded (FFPE) tissue samples from a single patient.

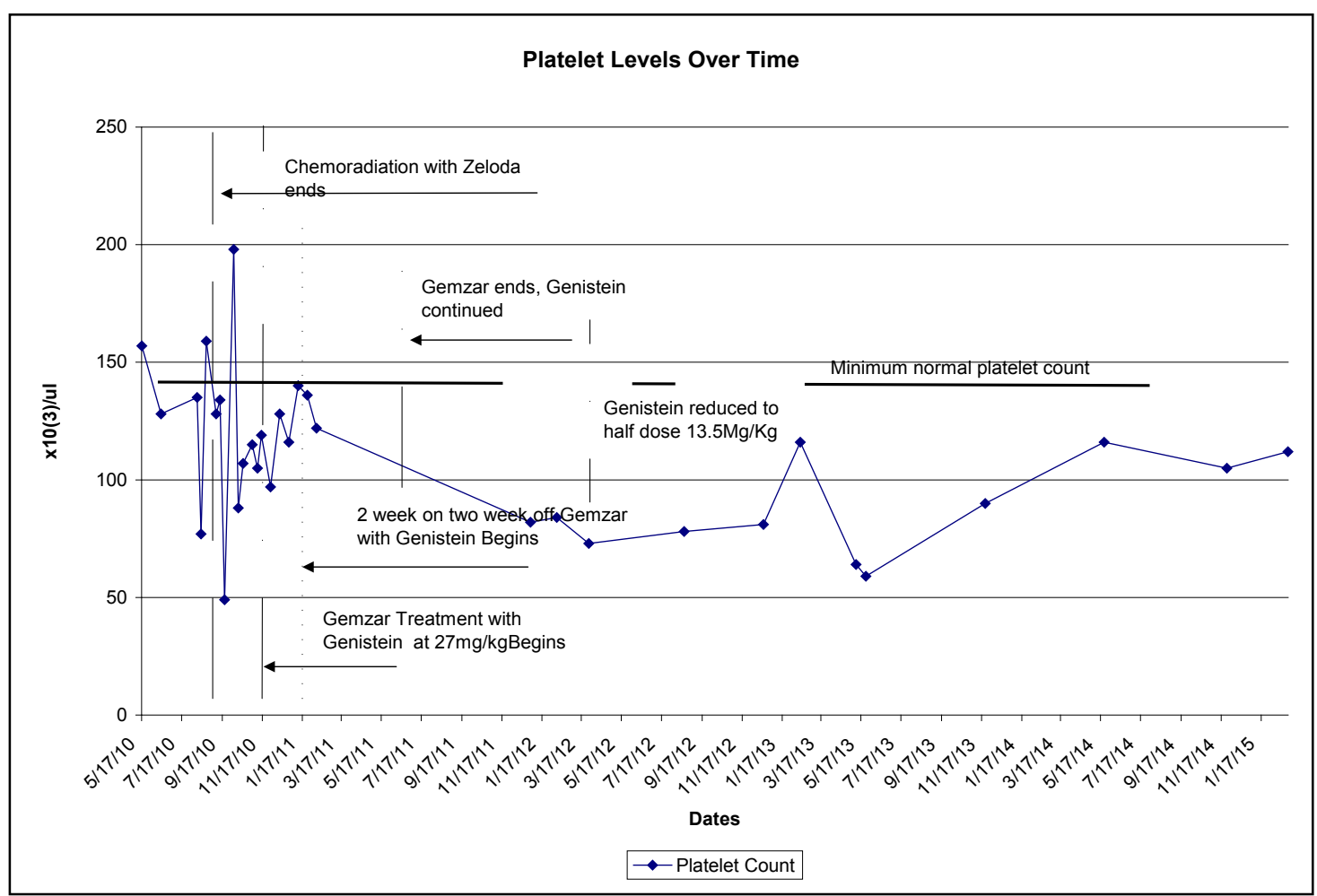

Figure 5. Correlation of chemotherapy/genistein regimen milestones and platelet levels derived from blood tests of single patient during adjuvant treatment.

rest period and installation of a sub-clavian power port for venous access, the Gemzar and genistein treatments were administered as described above. Oral genistein was initiated 48 hours before the initial Gemzar infusion.

Within three weeks of the start of phase two adjuvant treatments with Gemzar/genistein it was noted that the platelet count was reduced to less than 150,000 platelets per microliter, the low limit of the normal platelet count range in a healthy person. The treatment regimen was immediately modified to allow for recovery to satisfactory platelet levels, shifted from the standard three weeks on and one week off infusion regimen to a one week on infusion followed by a one week off infusion rest period.

The original Gemzar dosing schedule was planned to continue for three months but the change in infusion frequency and rest intervals extended the overall treatment time to six months. The high dose (16 $\mathrm{mg} / \mathrm{kg}$ of body mass divided over three doses per day) of genistein was continued throughout the period of Gemzar treatments.

There were no other noticeable side effects from the chemotherapy regimen and the patient remarked that she was expecting a much worse experience based on the side effects of the previous Zeloda/radiation treatment and its side effects. Whether the lack of side effects is attributable to the genistein or to some other cause will have to remain an observation without a suggested conclusion and be the starting point of further study, if needed. One anecdotal result of using genistein with chemotherapy regimens is that for some agents, including Gemzar, the side effects are lessened. Genistein is suspected of inhibiting the therapeutic effect of radiation and was therefore not used during that phase of the test. The patient in this study experienced no nausea, lethargy, hair loss, loss of appetite or serious gastric symptoms usually associated with this agent. 
Nehr AJ (2016) A single patient study of high dose isoflavones to enhance gemzar chemotherapy in post resection and post chemoradiation adjuvant treatment of pancreatic adenocarcinoma

Figure 5, below, is a chart of platelet levels and milestones during adjuvant treatment. The cause and effect relationship of the chemotherapy/genistein regimen on the platelet level as the dose and schedule of the Gemzar was adjusted is shown in Figure 4 as is the residual effect which demonstrates a slow recovery moving in the direction of normal in the last year shown. The net effect of the genistein cannot be separated from the chemotherapy agent except to the extent that the genistein dosage was consistent.

Perturbations are suspected to be related to Gemzar treatment and the adjustment of that agent alone. Overall, this chart represents the net effect of the combined treatment with a single adjustment in the Gemzar, as described.

\section{Non alcoholic fatty liver disease}

After the Whipple procedure, elevated levels of AST, ALT, and ALP are common. The elevation itself is expected but upward velocity is monitored for possible indication of disease recurrence.

This particular patient exhibited significantly elevated AST, ALT and ALP numbers on original presentation and the numbers failed to return to normal following surgery. Figures 5, 6, and 7 detail the histories of these elevated numbers. Several bouts of cholestasis were experienced, presumably due to fatty liver or to the adhesions detailed under the next heading. These inflammations drove CA19-9 and liver function metrics up, and caused malaise and symptoms of inflammation. The issues self resolved. This is not an unusual side effect of the Whipple procedure but must be monitored to ensure against liver damage and eventual cirrhosis.

\section{Liver adhesions and laparoscopic repair}

In response to an area of enhanced contrast in the upper portion of the liver, apparently becoming more pronounced, on a series of MRI studies, coupled with the unexplained and persistent elevated liver function tests, a laparoscopic examination was performed in August 2013. The examination revealed that the liver had adhered to the diaphragm over a large portion of the superior surface of the organ. The surgeon dissected the organ from the diaphragm and examined the areas of focal interest. A biopsy of the area of primary interest was found to consist of scar tissue and fatty deposition. No pathology suggesting malignancy was found. Multiple foci of enhanced contrast remained but none showed a cause for concern at the time of the procedure, four years postoperative.

\section{Liver function metrics}

At the time of the laparoscopic examination, four years post Whipple, all three liver functions under test (AST, ALT, ALP, Figures 5,6 , and 7 respectively) were trending back up after an eight month period of decline.

\section{Post whipple procedure charts}

It is not known if the levels for any of these liver functions observed are in any way affected by the high dose genistein regimen, per se. The complex relationship between the surgery and the resulting alteration to the digestive system make any conclusion tenuous at best. The patient developed multi focal fatty liver disease over the period of three years post operative, resulting in the decision to laparoscopically examine the liver. The fatty liver disease seems to have stabilized and the patient overall has remained healthy with no cirrhosis or return of malignancy. The conclusion at the time of this paper is that the liver dissection from the diaphragm done in August of 2013 eliminated the irritation caused by movement and the liver function numbers trended immediately in the direction of normal within months. The AST, ALT and ALP charts above, all show clear trends with AST only a few points within normal

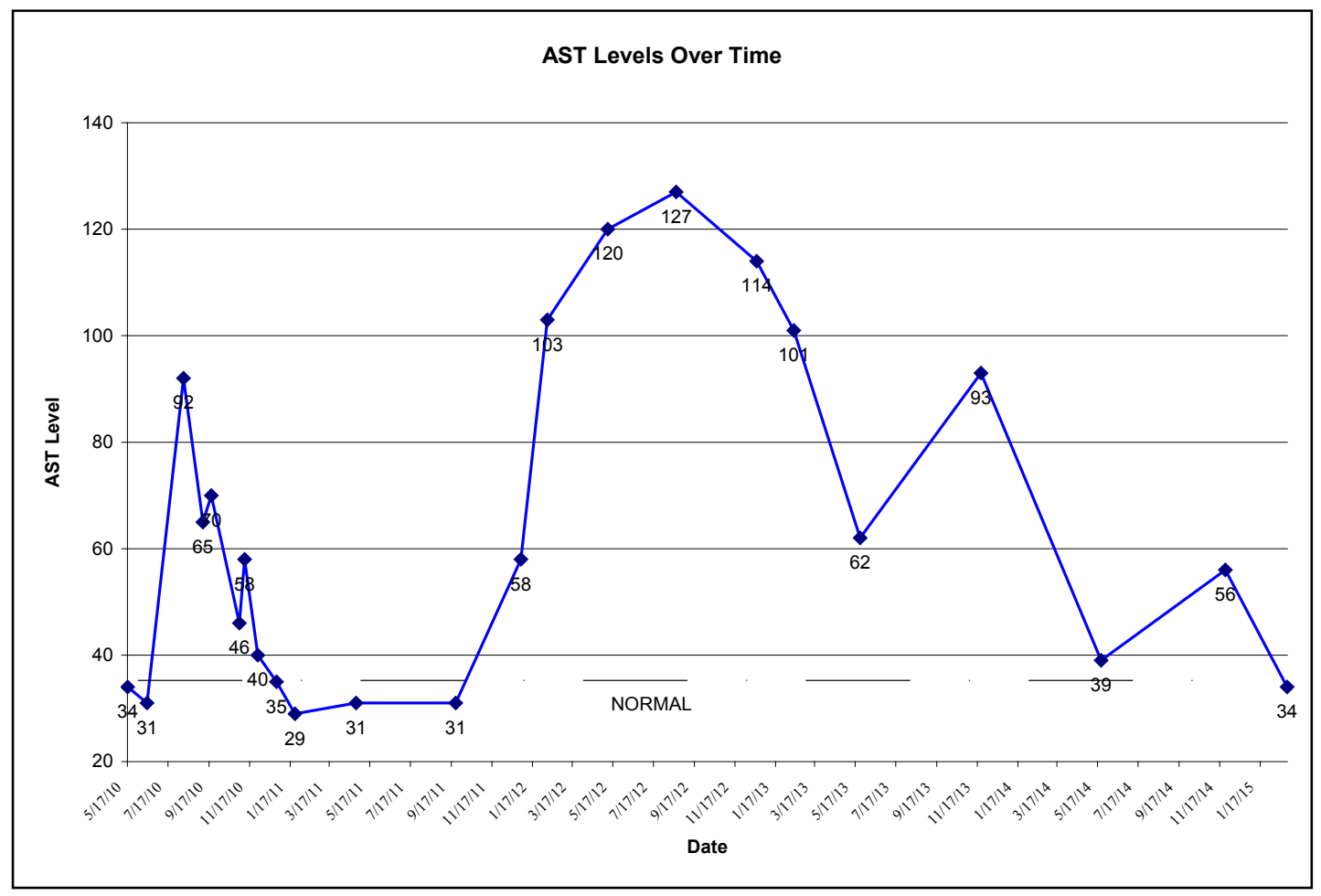

Figure 6. Single patient expression of aspartate aminotransferase (AST)derived from blood tests over a four year period subsequent to surgical tumor resection. 
Nehr AJ (2016) A single patient study of high dose isoflavones to enhance gemzar chemotherapy in post resection and post chemoradiation adjuvant treatment of pancreatic adenocarcinoma

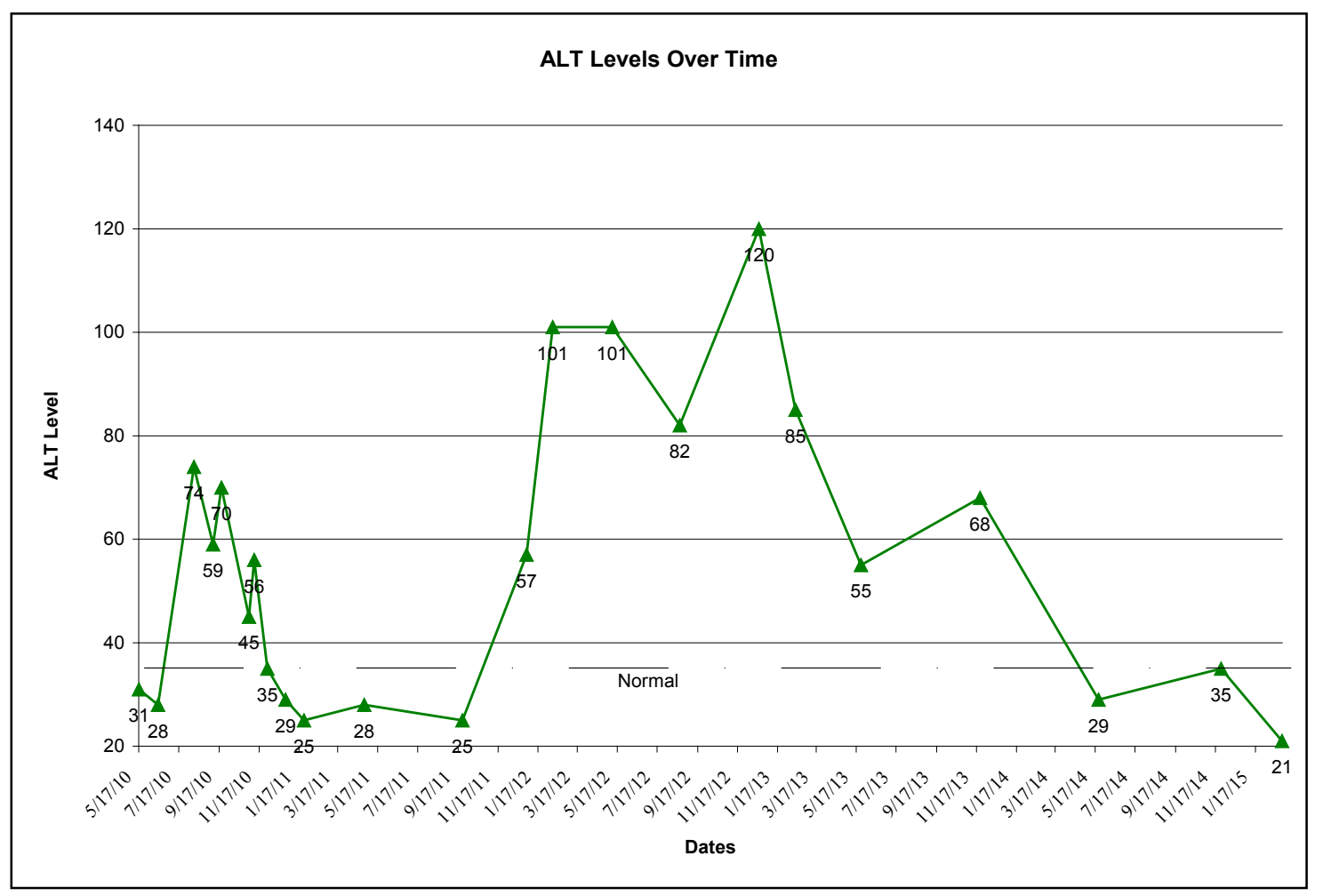

Figure 7. Single patient expression of alanine aminotransferase (ALT) derived from blood tests over a four year period subsequent to surgical tumor resection.

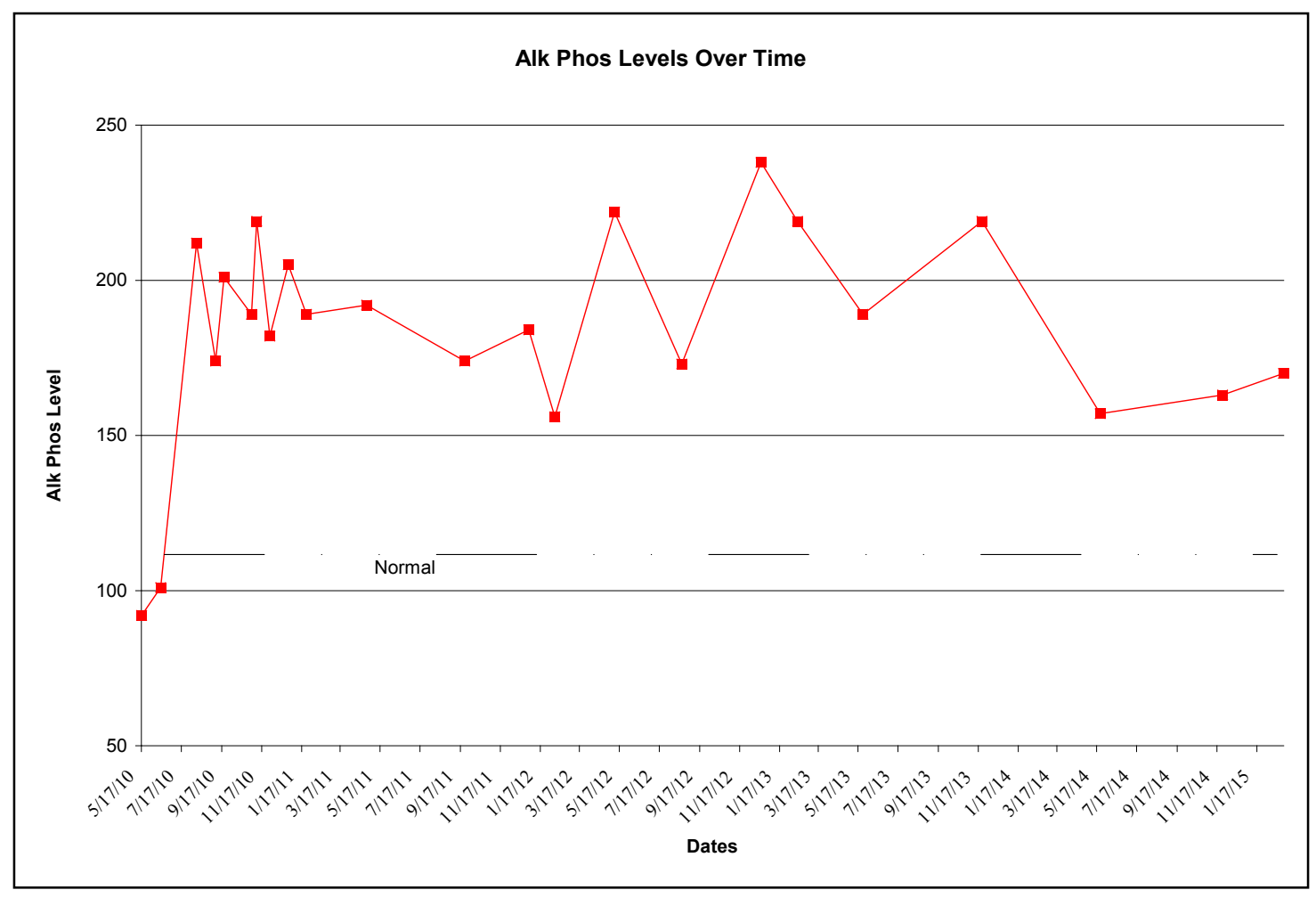

Figure 8. Single patient expression of alkaline phosphotase derived from blood tests over a four year period subsequent to surgical tumor resection. 
Nehr AJ (2016) A single patient study of high dose isoflavones to enhance gemzar chemotherapy in post resection and post chemoradiation adjuvant treatment of pancreatic adenocarcinoma

range, ALT well inside the normal range, and ALP rapidly trending in the direction of normal (Figures 6-8).

\section{Onset of diabetes}

Approximately one and one-half years post-Whipple procedure the patient experienced a slow but steady rise in blood glucose levels from a post operative range of $120-140$ to above 200. This required intervention, but the type of diabetes was difficult to determine. The patient had been mildly type 2 diabetic before surgery but diet controlled the issue and medication was not required. Post- Whipple procedure, the blood glucose was immediately elevated to 140 but stabilized at 120 and under, sometimes being normal (below 100). Eventually this changed and an upward trend was noticed, requiring the intervention of an endocrinologist. The type of diabetes was diagnosed to be insulin dependent.

Initially, an insulin regimen using Novalog and Levemir in combination for short and long action control, respectively, was instituted. The fast acting agent (Novalog) was eliminated because the patient experienced hypoglycemia upon administration of the standard dose for the level of blood glucose increase measured. Even lesser doses produced hypoglycemia and after this was revealed the Novalog was discontinued.

\section{Addition of metformin}

Metformin is known to not only be therapeutic for elevated blood glucose levels, but has also been suspected of inhibiting the growth of cancer stem cells, specifically adenocarcinoma stem cells $s^{6}$. These characteristics indicated that metformin could be efficacious for this patient but it was not considered at first in favor of the dual insulin regimen.

When the inclusion of Novalog in the treatment plan proved less than optimal, metformin was started and the Novalog discontinued. Levemir dosage at a set time every day was continued. The patient still experiences some variability in blood glucose levels but under this regimen it is controllable and is well tolerated.

\section{Ursodiol}

The persistent slow increase of fatty liver lesions and the slow but steady increase in AST, ALT and ALP metrics became increasingly worrisome to the treating physicians. An endoscopic retrograde cholangiopancreatography (ERCP) was performed and the determination was that while bile was flowing, the anatomy of the common bile duct was tortuous and not accessible for full examination. Eventually a specialist was consulted and he noted that a like symptomology is often seen in post-transplant liver patients. The standard treatment was to thin the bile with Actigall [7] (Ursodiol), a primary bile acid classically derived from ursine bile but now synthesized rather than harvested from animals. As can be seen on the AST, ALT and ALP charts the treatment has been successful in decreasing the liver metrics.

\section{Weight loss}

At the time of the cancer diagnosis the patient weighed $79.8 \mathrm{~kg}$ (176 pounds). As of this writing, five years later, she weighs $52.16 \mathrm{~kg}$ (115 pounds), a loss of $27.6 \mathrm{~kg}$ (61 pounds). Weight maintenance has been a persistent problem and is likely related to the change in her digestive system and the lessening of enzymes secreted by the pancreas, necessary for the absorption of nutrients. A regimen of Creon was begun and continued for two years but the proportion of protease, lipase and amylase left the patient unable to digest fats and unable to fine tune the enzymes needed to digest a variety of foods. A solution was found by buying all three components and taking them in various combinations depending on the meal. Patient comfort was greatly increased and weight loss velocity slowed and finally stabilized at 52.16 $\mathrm{kg}$. The dose of genistein has been modified periodically to reflect the changes in body mass. The patient continues to refine the method by which she self-medicates with enzymes, customized in ratio to the meal being consumed. Overall patient well being has increased steadily from the time of surgery to the date of this paper five years hence. Figure 9 graphically illustrates the timeline of the major treatment elements and the duration of the treatment and recovery from each element.

\section{Imaging studies history}

\section{Pre-surgical imaging studies}

Prior to engaging the diagnostic services of a high volume, research oriented, teaching facility, and in order of occurrence, the following studies were performed at a local hospital: a spiral CT scan, MRI without contrast, and endoscopic ultrasound. These studies revealed mild ductal dilation of the pancreatic duct and the extra-hepatic duct but were unrevealing of any definitive mass. Pancreatic cancer was suspected but not proved by the local physicians.

Upon transfer to the selected teaching facility, a second MRI, done with contrast, was performed and followed by a Spiral CT scan and EUS (Endoscopic Ultrasound), with stenting to relieve jaundice. Finally, an ERCP with needle biopsy was performed. Surgery was scheduled before the last two studies were complete due to the strong indication that laparoscopic intervention would be required for any of the possibilities that were suggested by the findings so far. The biopsy, taken just two days earlier proved to be revealing and the definitive diagnosis of adenocarcinoma was delivered to the surgeon just prior to surgery.

\section{Post-surgical imaging studies}

Subsequent to the surgery, most imaging has been by MRI;

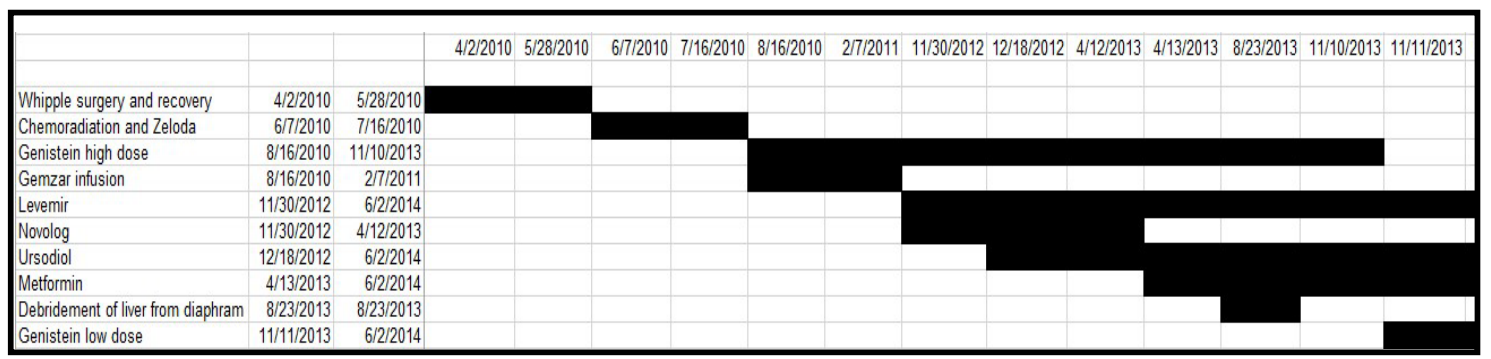

Figure 9. Timeline of major treatment elements administered to the single patient subject, providing initial treatment dates and either recovery period or ending treatment dates, as appropriate. 
the frequency coincided with the data points shown in the miR-21 expression data plot because the blood for miR-21 was drawn close to the time of each imaging study. Since the Whipple surgery, and aside from the slow progression and eventual stabilization of the fatty liver deposits, no other areas of focal interest have been observed.

The patient was on a six month recurrent examination cycle from surgery to 2012 and was put on a three month cycle while the fatty liver problem was addressed. As of late 2013 she was returned to the six month cycle.

\section{Discussion and conclusion}

Drawing any firm conclusion from a study where $\mathrm{N}=1$ is scientifically tenuous at best, yet there are some significant aspects of this study that warrant further investigation despite the single patient limitation. The percentage of survival at the five year point for all resection candidates is $25 \%$ at best $[1,6]$ and is reliant on several factors, all of which were present in this case. The fact that the disease was localized, did not invade the retroperitonial wall, did not involve the mesenteric artery or the portal vein, did not spread to the lymph nodes and was sized at $2 \mathrm{~cm}$ all favored this outcome. Further choices were made, consistent with one of the cited papers [1] that also enhanced statistical survivability. A teaching hospital with a highly experienced chief pancreatico-biliary surgeon [1] was sought and he, in turn, used minimally invasive techniques to limit blood loss and to speed recovery so that adjuvant treatment would occur without delay and not be impacted by recovery time due to post operative complications. Even with these factors this patient presented an anomalous and interesting recovery. To date, she feels fully recovered and states a $90 \%$ quality of life metric by self assessment. Her CA19-9 and miR-21 levels are both in the normal range which is somewhat unusual for CA19-9 and very unusual for miR-21 expression. A study of post-resection patients and the miR-21 paper cited below [7] both suggest that a factor of several multiples of normal would be expected in the entire post resection period. The studies were done by experienced teaching institutions and adjuvant protocols were followed. The only differential between the patients in the cited studies and the one in this study was the administration of isoflavones (genistein), in accordance with the cited reference [3], which was administered immediately prior to, during, and after, the cycle of Gemzar chemotherapy. The miR-21 declined to previously unseen levels and remained there except for the two periods of liver inflammation which elevated it, along with the CA19-9 levels, but with a lead-lag time offset. The significant metric is that the levels remained uncharacteristically low and the patient remained disease free for the three year period after surgery and chemotherapy. The isoflavones were continued at half dose levels and adjusted for body mass changes from six months after the cessation of Gemzar to the end of the study period and is expected to continue for the rest of the patient's life. This continuation is theorized to continue suppressing the NF- $\kappa B$ pathway and thus inhibit disease recurrence. Additionally metformin was added two years post operatively when the miR-21 levels were already uncharacteristically low. However, this agent cannot be ignored because it is known to inhibit malignant stem cell growth [6], so while it cannot be credited with the impressively low miR-21 level, which manifested and continued before the addition of metformin to the treatment regimen, it may be adding to disease inhibition.

Also of note is the ease with which the patient experienced the entire surgery, treatment, and recovery process. Side effects of the surgery were minimal and hospital release occurred in six days. Side effects from the radiation/Zeloda phase were as expected and presented the only problem areas for this patient. Side effects from the Gemzar/genistein phase were far less than expected and were easily tolerated. The side effects of all subsequent treatments have been easily manageable. Oily discharge at stool is the primary complaint and is managed easily with a regimen of lipase, protease and amylase supplements taken with the meal, depending on the composition of the food being consumed. The outcome of this case is highly unusual in the aspect of the low miR-21 expression, as well as the ease with which the patient recovered and the quality of life restored. The prognosis remains excellent with the very real and unusual expectation of complete cure being discussed. This study, while limited to one patient, should provide reason to further study the use of genistein in humans with resectable disease during the Gemzar phase and later to attempt to duplicate the same result in a much larger population. Also the metric of miR-21 level seems to be somewhat revealing of efficacy of the adjuvant treatment and also seems very reactive to non-metastatic liver complications. This too should be investigated further.

While it is possible that the surgical intervention and adjuvant treatment alone could have produced the survival seen (in fact that is true in $25 \%$ of post resection cases) the anomalous miR-21 expression level and the robust recovery of this particular patient are both highly unusual even within that population. It is consistent with the observations of this patient to conclude 1) the obvious; that this course of treatment was not harmful and did not cause recurrence, and 2) that the presentation of this patient is consistent with the hypothesis in the paper cited by Banerjee et al. [3] and inconsistent with the paper cited by Ali et al. [8] regarding the expected miR-21 levels of pancreatic cancer patients both resectable and unresectable. The majority of the patients in that study experienced recurrent disease or remained unresectable and all but three had much higher miR-21 levels; none had equivalent or lower levels than the patient in the current study. All succumbed to the disease within a short period of time. The entire group had a mean survival of seven months. The patient in the single patient study exhibited very low miR-21 expression level, and survived far longer than any of the 50 subjects in the above paper. She remains disease free long after the time when all of the study participants in the referenced works had died, and has recovered to a high quality of life. She has become active in furthering the research into this disease by using her energy to advocate for increased research into this disease. She travels frequently and has returned to vital engaged life!

Further study of the effect of genistein in increasing Gemzarinduced apoptosis, and on interrupting the Notch/ NF- $\kappa B$ pathway [9], study of the significance and limitations of miR-21 expression in predicting survival and chemoeffectiveness, and study into the role metformin plays in pancreatic cancer treatment are all needed. This disease has a dismal prognosis in the vast majority of cases. That it did not have a tragic outcome in this case is, by itself, significant. This patient incurred risk to provide the limited data presented here and that contribution must not be wasted.

\section{Acknowledgements}

Much good must be said about the team of doctors and researchers who performed the research that led to this experiment. Also, upon patient request Dr. Fazlul Sarkar and Ms.Shadan Ali volunteered and performed the expression of miR-21levels in plasma as well as tumor tissue that provided the data on those metrics in the paper. The treatment team also cannot be overlooked. Dr Cecilia Pineda, the patient's primary care physician who first discovered the abnormal blood results and took immediate action to investigate, Dr. Robert 
Nehr AJ (2016) A single patient study of high dose isoflavones to enhance gemzar chemotherapy in post resection and post chemoradiation adjuvant treatment of pancreatic adenocarcinoma

Dorff who first suspected the disease was cancer rather than a gall bladder issue. The diagnostic and surgery team under Dr. Horacio Asbun at Mayo Clinic, Jacksonville, FL who confirmed the disease and performed the surgery, the oncology team comprised of Dr. Omar Kayaleh and Karen Przystup at MD Anderson Cancer Center, Orlando, FL who provided oncology support and administered the Gemzar phase of the treatment with the genistein as an enhancement at patient request, and Dr. Daniel Buchholz, MD Anderson Cancer Center, Orlando, FL. who administered the targeted radiation phase.

Review and advice for this paper was provided by Ms Shandan Ali, Dr. Fazlul Sarkar, Dr. Howard Crawford, erstwhile Associate Dean and Pancreatic Cancer researcher at Mayo Clinic, Jacksonville, FL. Dr. Crawford provided review of this paper at the author's request, as did Dr. Andrew C. Schuerger from University of Florida.

\section{References}

1. Lim JE, Chien MW, Earle CC (2003) Prognostic Factors Following Curative Resection for Pancreatic Adenocarcinoma A Population Based, Linked Database Analysis of 396 Patients. Ann Surg 237: 74-85. [Crossref]
2. Shishodia S,Aggarwal BB (2002) Nuclear Factor-kappaB Activation: A Question of Life or Death. JBiochemMolBiol35: 28-40. [Crossref]

3. Banerjee S, Zhang Y, Ali S, Bhuiyan M, Wang Z, et al. (2005) Molecular Evidence for Increased Antitumor Activity of Gemcitabine by Genistein In vitro and In vivo Using an Orthotopic Model of Pancreatic Cancer. Cancer Res 65: 9064-9072. [Crossref]

4. Arlt A, Schäfer H, Kalthoff H (2012) The 'N-factors' in pancreatic cancer: functional relevance of NF-kB, NFAT and Nrf2 in pancreatic cancer. Oncogenesis1: e35. [Crossref]

5. Badger SA, Brant JL, Jones C, McClements J, Loughrey MB, et al. (2010) The role of surgery for pancreatic cancer: a 12-year review of patient outcome. Ulster Med $\mathrm{J} 79$ : 70-75. [Crossref]

6. Kisfalvi K, Eibl G, Sinnett-Smith J, Rozengurt E (2009) Metformin disrupts crosstalk between $\mathrm{G}$ protein-coupled receptor and insulin receptor signaling systems and inhibits pancreatic cancer.CancerRes 69: 6539-6545. [Crossref]

7. http://en.wikipedia.org/wiki/Ursodiol

8. Ali S, Almhanna K, Chen W, Philip PA, Sarkar FH (2010) Differentially expressed miRNAs in the plasma may provide a molecular signature for aggressive pancreatic cancer. Am J Transl Res 3: 28-47. [Crossref]

9. Wang Z, Ahmad A, Li Y, Azmi AS, Miele L, et al. (2011) Targeting notch to eradicate pancreatic cancer stem cells for cancer therapy. Anticancer Res 31: 1105-1113. [Crossref]

Copyright: (C) 2016 Nehr AJ. This is an open-access article distributed under the terms of the Creative Commons Attribution License, which permits unrestricted use, distribution, and reproduction in any medium, provided the original author and source are credited. 